\title{
紫细菌光合反应中心电子激发态的理论研究 ${ }^{*}$
}

\author{
任彦亮 程 林 万 坚 ${ }^{* *}$ 李永健 刘俊军 \\ 杨光富 $^{* *}$ 张丽华 杨 嵩
}

(华中师范大学化学学院 农药与化学生物学教育部重点实验室, 武汉 430079)

\begin{abstract}
摘要 运用 ZINDO 和 INDO/S 两种量子化学理论方法, 对紫细菌(Rhodopseudomonas (Rps.) viridis) 光合反应中心色素分子簇的电子激发态进行了理论研究. 通过理论计算的电子激发态光 谱与实验吸收光谱, Circular Dichroism 光谱的比较, 对实验光谱重新进行了较为合理的归属. 考 察了色素分子间相互作用、色素分子与蛋白质中氨基酸残基相互作用对激发态光谱的影响. 研究 结果表明: Rps. viridis 光合反应中心色素分子簇间的相互作用以及氨基酸残基与色素分子的作用, 对激发态光谱的性质具有一定的影响; 不考虑色素分子间相互作用以及色素分子与周围氨基酸 残基的作用，只基于单个色素分子(或其模型分子)理论计算进行光谱归属是不合理的.
\end{abstract}

\section{关键词 紫细菌(Rps. viridis) ZINDO INDO/S Circular Dichroism 光谱}

光合作用是地球上最重要的化学反应之一, 其 对光能转化为化学能的快速, 高效以及潜在的应用 前景使人们期望对其各种能量传递及电子转移机理 获得更深层的认识. 光合反应中心(Photosynthetic Reaction Center (PSRC)) 是光合作用的核心部分, 作 为研究光合作用的切入点, 已成为国内外众多理论 和实验工作者最为关注的研究对象. 但是, 直到 20 世纪末, Deisenhofer等人首次报道了紫细菌Rps. viridis的X射线晶体结构 ${ }^{[1]}$, 人们对光合反应中心的研究 才进入了原子-分子水平.

紫细菌(Rps. viridis)光合反应中心 ${ }^{[2]}$ 包含 4 个细
菌叶绿素分子Bacteriochlorophyll b(Bcl), 其中两个通 过强烈的耦合形成所谓的“特殊对”(P), 另外两个 Bcl 分别排列在 $P$ 的两侧, 通常称之为辅助细菌叶绿素 $(\mathrm{ABcl})$, 简写为 $(\mathrm{B})$, 在 $\mathrm{ABcl}$ 的下面分别有两个细菌 去镁叶绿素分子Bacteriopheophytin $\mathrm{b}(\mathrm{Bpb})$, 简写为 $(\mathrm{H}), \mathrm{Bpb}$ 下面是两个醌分子 Ubiquinone (UQ) 和 Menaquinone (MQ), 两个醌分子中间有一个非血红 素二价铁离子, 如图 1 所示. 这些色素分子沿“特殊 对” $P$ 两侧的排列, 具有准 $C_{2}$ 对称性 (一般以 $P$ 的两个 $\mathrm{Mg}$ 原子连线的中点与 $\mathrm{Fe}$ 原子的连线作为 $\mathrm{C}_{2}$ 轴), 距 Rps. viridis反应中心的 $\mathrm{M}$ 链较近的一侧为 $\mathrm{M}$ 分支, 而

* 国家自然科学基金(批准号: 20203009, 20432010)、湖北省自然科学基金创新群体项目(批准号: 2004ABC002)、国家重大基础研究前期专项 (批准号: 2004CCA00100)和湖北省青年杰出人才基金及教育部留学回国人员启动基金资助项目

**联系人, E-mail: jianwan@mail.ccnu.edu.cn, gfyang@mail.ccnu.edu.cn 
距 $L$ 链较近的一侧则为 $L$ 分支. 但与上述准 $C_{2}$ 轴对称 性不同的是: 研究发现, 当紫细菌反应中心的原初电 子供体 $\mathrm{P}$ 受到光激发变成单线垂直激发态后, 被激发 的电子只沿着 $\mathrm{L}$ 分支到达 $\mathrm{UQ}^{[3,4]}$, 而在 $\mathrm{M}$ 分支则几乎 检测不到电子转移的发生, 为了对上述电子转移的 机理获得深入的了解, 其电子激发态的计算和合理 归属就成了最重要的环节之一. 由于紫细菌的PSRC 是个巨大的分子体系(加氢后近 1000 个原子), 若采用 对称性匹配电子耦合相关簇组态相互作用 (symmetry-adapted cluster configuration interaction (SAC-CI)) 方法 ${ }^{[5 \sim 7]}$ 、时间相关的密度泛函理论方法 (time-dependent density functional theory (TDDFT) $)^{[8,9]}$ 、多组态二级微扰理论方法(multiconfigurational second-order perturbation theory (CASPT2) $)^{[10]}$ 以及运动方程耦合簇方法(equation-of-motion coupled cluster(EOM-CC) $)^{[11]}$ 等Post-SCF理论计算方法对整个 反应中心直接进行计算, 就目前计算资源来说是难 以实现的.

随着计算机科学的飞速发展和一些新的算法的 提出, 人们可以对单个色素分子的模型分子进行激 发态光谱的理论计算 ${ }^{[12 \sim 17]}$, 例如Hasegawa等人 ${ }^{[13]}$ 采 用SAC-CI方法对Rps. Viridis光合反应中心单个色素 分子的模型化分子(将色素分子的支链去掉, 一些取 代基用氢原子代替, 只保留环状共轭体系)进行了激 发态光谱的计算. SAC-CI方法对较为适中分子体系 激发态光谱的计算具有很高的精度 ${ }^{[5 \sim 7]}$, 但是对 Rps. viridis光合反应中心这样大的生物分子体系, SAC-CI 计算目前只能限于单个色素分子的模型化分子. 基 于单个色素分子的模型的弱点在于不能考虑各色素 分子间的相互作用以及色素分子与周围氨基酸残基 的相互作用对激发态光谱的影响.

近年来, 随着半经验分子轨道理论方法 Intermediate Neglect of Differential Overlap $(\mathrm{INDO} / \mathrm{S})^{[18]}$ 和Zerner's INDO/1 (ZINDO) $)^{[19]}$ 的经验参 数的更加合理化和精确化, 其对低能量激发态理论 光谱的研究已经具有较高的精度, 并且优点在于可 以应用到相当大的生物分子体系. 本文将运用这两 种方法对Rps. viridis光合反应中心的完整色素分子簇 的电子激发态进行理论研究, 并对实验光谱重新进
行归属. 本文的主要目的是通过对基于PSRC分子簇 模型的ZINDO和INDO/S计算结果与实验吸收光谱 [20]、实验Circular Dichroism (CD)光谱 ${ }^{[21]}$ 以及基于单 个色素分子模型的SAC-CI计算结果 ${ }^{[17]}$ 进行比较研究 来证明: 色素分子间的相互作用以及周围氨基酸残 基与色素分子的相互作用对理论计算光谱会产生一 定的影响. 为此, 本文计算研究中同时考虑了色素分 子簇以及距色素分子簇 $2.5 \AA$ 范围内所有氨基酸残基 分子. 与特殊对 P作用较强的有组氨酸 HIS173 和 HIS 200, 这两个组氨酸分别与 $\mathrm{P}_{M}$ 和 $\mathrm{P}_{\mathrm{L}}$ 的镁原子形成 轴向配位键. 组氨酸HIS 180 和HIS 153 分别与 $\mathrm{B}_{\mathrm{M}}$ 和 $\mathrm{B}_{\mathrm{L}}$ 的镁原子形成轴向配位键. 在MQ和UQ中间, 有 4 个 组氨酸HIS190, HIS217, HIS230, HIS264 和 1 个谷氨酸 GLU232 与铁原子有一定的作用, HIS2 17 与MQ的距离 较近, 可能形成氢键, HIS190 可能与UQ形成氢键, 如 图 1 所示. 具体计算细节将在下面部分介绍.

\section{1 计算方法}

Rps. Viridis光合反应中心色素分子簇是个较大 的超分子体系, 由于目前计算条件的限制, 对其结构 优化较为复杂, 而且优化结构的合理性较难验证, 晶 体结构相对合理, 因此, 本文计算主要基于蛋白质数 据库中的 1 PRC的晶体数据 ${ }^{[2]}$, 用Gaussian view 3.07 添加所有的氢原子。保持非氢原子坐标不变, 用 Gaussian $03^{[22]}$ 对所有的氢原子作平衡态几何构型的 部分优化, 使氢原子坐标更为合理. 考虑到氢原子坐 标的合理性可能会影响整个光谱性质, 我们对Rps. viridis 光合反应中心的氢原子同时用 Parametric Model number 3 (PM3) ${ }^{[23]}$ 和 Universal Force Field (UFF $)^{[24]}$ 两种方法作了部分优化. 结果显示, 基于半 经验分子轨道方法PM3 的优化结构仅使UV光谱能带 发生了 $0.01 \mathrm{eV}$ 的蓝移，但用PM3 方法计算耗时 7 天 的任务对于UFF方法只需要 $40 \mathrm{~s}$, 因此本文所用晶体 结构的氢原子坐标均为UFF力场优化的结果. 激发态 能量和振子强度的计算采用INDO/S和ZINDO. 计算 比较发现, 加上UQ和MQ两个色素分子后 $2.0 \mathrm{eV}$ 之前 的能带几乎没有变化，因此，本文的计算中只把原初 电子供体 $\mathrm{P}, \mathrm{M}$ 分支的 $\mathrm{B}_{\mathrm{M}}, \mathrm{H}_{\mathrm{M}}$ 和L分支的 $\mathrm{B}_{\mathrm{L}}, \mathrm{H}_{\mathrm{L}}$ 五个色 素分子作为一个分子簇进行计算. 


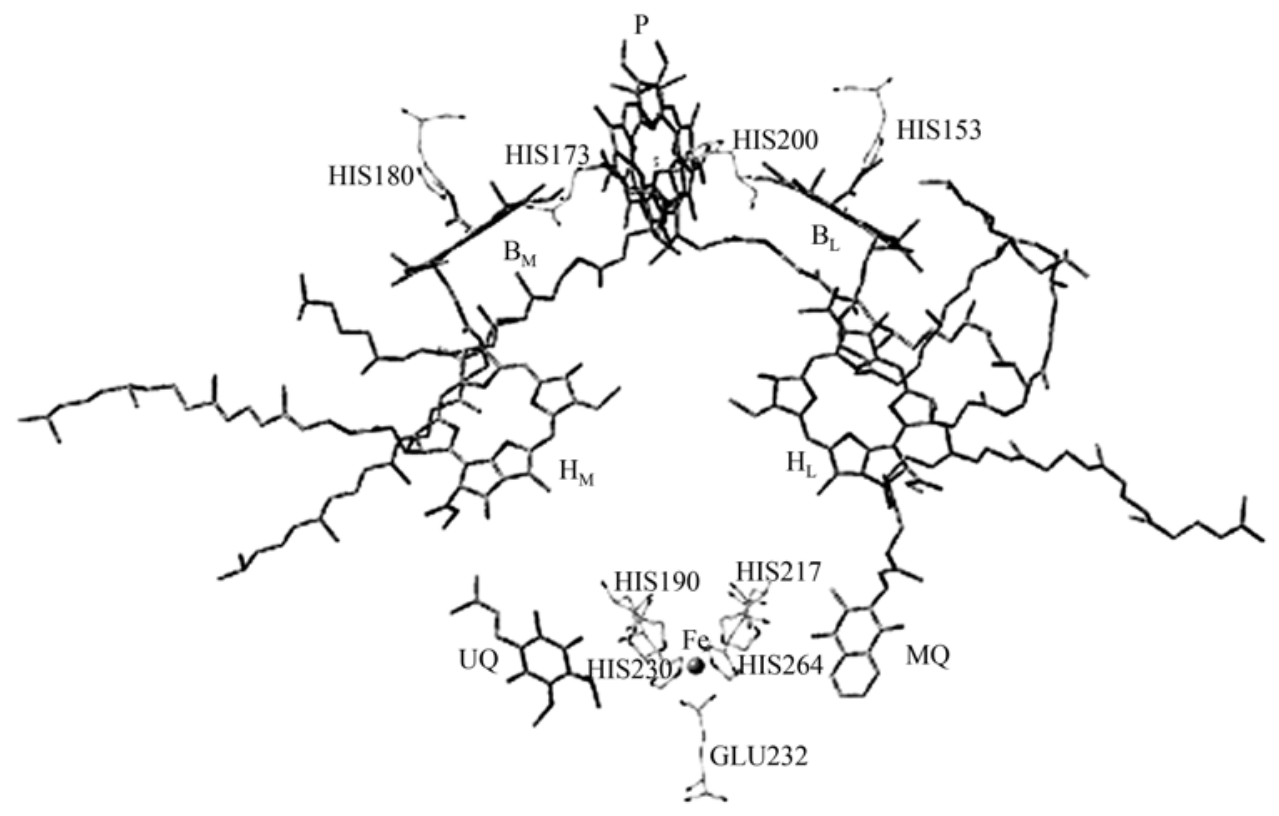

图 1 紫细菌(Rps. viridis)反应中心中各色素分子的结构

在 $\mathrm{CD}$ 光谱的计算中, 横坐标为激发态能量 (excited energy), 纵坐标为旋光强度 (rotatory strengths (velocity)), 简称 R. ZINDO 的计算是用 SGI/Origin 300 服务器上的Gaussian 03 软件包完成 ${ }^{[22]}$, INDO/S计算用的是MOSF程序 ${ }^{[25]}$.

\section{2 结果与讨论}

\section{1 激发态光谱的归属}

通过对 $P, B_{L}, B_{M}, H_{L}, H_{M}$ 色素分子簇的自洽场计 算发现, 体系的分子轨道大部分都定域到某个色素 分子上, 表 1 列出了前 6 个最高占据轨道(HOMO)和 最低空轨道(LUMO)的分子轨道能量及其在色素分子 上的定域情况.

考虑到半经验方法一般对低能量带光谱有较好 预测 ${ }^{[26]}$, 本文分别采用ZINDO和INDO/S两种方法, 对紫细菌光合反应中心低能量区域的激发态 $(<2.0 \mathrm{eV})$ 进行了理论计算, 计算结果如表 2 和图 2 所示. 由表 2 可见 $1^{1} A$ 激发态的相关组态主要是 $H \rightarrow L+1$, 且由表 1 可知 $\mathrm{H}, \mathrm{L}+1$ 两个分子轨道主要为色素 $\mathrm{P}$ 的贡献, 因 此我们将 $1^{1} A$ 激发态归属为 $\mathrm{P}$ 自身电子的激发. 另外,
从图 3(c)中可见 $1^{1} A$ 激发态的旋光强度为正, 说明其 跃迁矢量与 $\mathrm{C}_{2}$ 轴垂直, 用 $\mathrm{P}_{\mathrm{y}}$ 表示. $2^{1} A$ 激发态的相关 组态主要是 $\mathrm{H}-2 \rightarrow \mathrm{L}+1$ 和 $\mathrm{H}-3 \rightarrow \mathrm{L}+1$, 由表 1 可知, 特 殊对 $\mathrm{P}$ 与辅助细菌叶绿素 $\mathrm{B}_{\mathrm{L}}$ 对 $\mathrm{H}-2$ 分子轨道的贡献 分别为 0.521 和 0.478 , 对 H-3 分子轨道的贡献分别为 0.477 和 0.522 , 由此可见, 由于特殊对 $\mathrm{P}$ 与辅助细菌 叶绿素 $B_{L}$ 的较强的相互作用而导致 H-2 和 H-3 分子

表 1 紫细菌光合反应中心中各色素分子对整个体系分子 轨道的贡献 ${ }^{\text {a) }}$

\begin{tabular}{ccccccc}
\hline 分子轨道 & 能量/au & $\mathrm{P}$ & $\mathrm{B}_{\mathrm{M}}$ & $\mathrm{B}_{\mathrm{L}}$ & $\mathrm{H}_{\mathrm{M}}$ & $\mathrm{H}_{\mathrm{L}}$ \\
\hline $1045(\mathrm{HOMO}-5)$ & -0.23419 & & & & & 0.999 \\
$1046($ HOMO-4) & -0.23127 & & & & 0.999 & \\
1047 (HOMO-3) & -0.22329 & 0.477 & & 0.522 & & \\
$1048($ HOMO-2) & -0.22311 & 0.521 & & 0.478 & & \\
1049 (HOMO-1) & -0.21908 & & 0.997 & & & \\
$1050(\mathrm{HOMO})$ & -0.2173 & 0.997 & & & & \\
$1051(\mathrm{LUMO})$ & -0.0932 & & & & & 0.998 \\
$1052($ LUMO+1) & -0.09162 & 0.999 & & & & \\
$1053($ LUMO+2) & -0.08613 & & & & 0.999 & \\
$1054($ LUMO+3) & -0.0841 & & & 0.998 & & \\
$1055($ LUMO+4) & -0.07908 & & 0.999 & & & \\
$1056($ LUMO+5) & -0.07354 & 0.999 & & & & \\
\hline
\end{tabular}

a) 只列出贡献大于 0.05 的数据 
表 2 Rps. viridis 光合反应中心中各色素分子激发态能量的理论计算与实验值的比较

\begin{tabular}{|c|c|c|c|c|c|c|c|c|c|c|c|c|c|}
\hline \multirow{2}{*}{ 发色团 } & \multirow{2}{*}{$\begin{array}{c}\text { 激发 } \\
\text { 态 }\end{array}$} & \multicolumn{4}{|c|}{$\operatorname{ZINDO}(1)^{\mathrm{a})}$} & \multicolumn{2}{|c|}{$\operatorname{ZINDO}(2)^{\mathrm{b})}$} & \multicolumn{2}{|c|}{$\operatorname{INDO} / \mathrm{S}(1)^{\mathrm{a})}$} & \multicolumn{2}{|c|}{$\operatorname{INDO} / \mathrm{S}(2)^{\mathrm{b})}$} & \multicolumn{2}{|c|}{$\begin{array}{l}\text { 实验值 } \\
{[20,21]}\end{array}$} \\
\hline & & 相关组态 ${ }^{c)}(|c|>0.3)$ & $\begin{array}{l}\text { 激发 } \\
\text { 能/eV }\end{array}$ & $\begin{array}{c}\text { 振子强 } \\
\text { 度 }\end{array}$ & $\begin{array}{l}R / 10^{-40} \\
\operatorname{cgs}\end{array}$ & $\begin{array}{c}\text { 激发能 } \\
/ \mathrm{eV}\end{array}$ & 振子强度 & 激发能/eV & $\begin{array}{c}\text { 振子强 } \\
\text { 度 }\end{array}$ & $\begin{array}{c}\text { 激发能 } \\
/ \mathrm{eV}\end{array}$ & $\begin{array}{l}\text { 振子强 } \\
\text { 度 }\end{array}$ & $\begin{array}{l}\text { 激发 } \\
\text { 能/eV }\end{array}$ & 峰 \\
\hline $\mathrm{P}_{\mathrm{y}}$ & $1^{1} A$ & $0.59(\mathrm{H} \rightarrow \mathrm{L}+1)$ & 1.17 & 0.951 & 85.8 & 1.22 & 0.905 & 1.40 & 1.223 & 1.43 & 1.120 & 1.25 & I \\
\hline $\mathrm{P}_{\mathrm{x}} / \mathrm{B}_{\mathrm{L}} \rightarrow \mathrm{P}$ & $2^{1} \mathrm{~A}$ & $\begin{aligned} 0.35(\mathrm{H}-2 & \rightarrow \mathrm{L}+1)-0.33(\mathrm{H}-3 \\
& \rightarrow \mathrm{L}+1)\end{aligned}$ & 1.32 & 0.502 & -80.1 & 1.32 & 0.419 & 1.57 & 0.724 & 1.57 & 0.618 & 1.46 & II \\
\hline $\mathrm{H}_{\mathrm{L}}$ & $3^{1} \mathrm{~A}$ & $0.56(\mathrm{H}-5 \rightarrow \mathrm{L})$ & 1.36 & 0.276 & 5.2 & 1.35 & 0.417 & 1.61 & 0.257 & 1.59 & 0.513 & 1.49 & IV \\
\hline $\mathrm{B}_{\mathrm{M}}$ & $4^{1} \mathrm{~A}$ & $0.55(\mathrm{H}-1 \rightarrow \mathrm{L}+4)$ & 1.41 & 0.589 & -79.7 & 1.38 & 0.329 & 1.65 & 0.602 & 1.64 & 0.323 & 1.46 & II \\
\hline $\mathrm{B}_{\mathrm{L}} / \mathrm{P} \rightarrow \mathrm{B}_{\mathrm{L}}$ & $5^{1} A$ & $\begin{aligned} 0.40(\mathrm{H}-3 & \rightarrow \mathrm{L}+3)+0.39(\mathrm{H}-2 \\
& \rightarrow \mathrm{L}+3)\end{aligned}$ & 1.43 & 0.126 & 46.0 & 1.42 & 0.156 & 1.69 & 0.340 & 1.69 & 0.331 & 1.49 & III \\
\hline $\mathrm{H}_{\mathrm{M}}$ & $6^{1} \mathrm{~A}$ & $0.61(\mathrm{H}-4 \rightarrow \mathrm{L}+2)$ & 1.47 & 0.305 & -3.2 & 1.48 & 0.364 & 1.72 & 0.241 & 1.72 & 0.363 & 1.57 & V \\
\hline
\end{tabular}

a) 不考虑光合反应中心周围的氨基酸; b)考虑与光合反应中心距离为 $2.5 \AA$ 之内的氨基酸; c)H, L 分别代表 HOMO, LUMO

轨道由发色团 $\mathrm{P}$ 和 $\mathrm{B}_{\mathrm{L}}$ 线性组合而成. $\mathrm{L}+1$ 分子轨道主 要为发色团 $\mathrm{P}$ 的贡献，因此 $2^{1} A$ 激发态主要来自特殊 对 $\mathrm{P}$ 的激发和 $\mathrm{B}_{\mathrm{L}} \rightarrow \mathrm{P}$ 的电荷转移(charge transfer (CT)) 的贡献. 由于 $2^{1} A$ 激发态的旋光强度 $(R)$ 为负, 说明其 跃迁矢量与准 $\mathrm{C}_{2}$ 轴平行, 因此, 我们归属 $2^{1} A$ 态为 $\mathrm{P}_{\mathrm{x}} / \mathrm{B}_{\mathrm{L}} \rightarrow \mathrm{P}$ 激发态. $3^{1} A$ 激发态的相关组态主要是 $\mathrm{H}-5 \rightarrow \mathrm{L}, \mathrm{H}-5$ 和 $\mathrm{L}$ 分子轨道均来自于色素 $\mathrm{H}_{\mathrm{L}}$ 的主要贡 献，因此 $3^{1} A$ 激发态归属为 $\mathrm{H}_{\mathrm{L}}$ 的电子激发. $4^{1} A$ 激发 态的相关组态是 $\mathrm{H}-1 \rightarrow \mathrm{L}+4$, 由表 1 可知, $\mathrm{H}-1$ 和 $\mathrm{L}+4$ 分子轨道主要来自于色素 $\mathrm{B}_{\mathrm{M}}$ 的贡献, 因此, 我们将 $4^{1} A$ 激发态归属为 $\mathrm{B}_{\mathrm{M}}$ 的电子激发. $5^{1} A$ 激发态的相关 组态主要是 $\mathrm{H}-3 \rightarrow \mathrm{L}+3$ 和 $\mathrm{H}-2 \rightarrow \mathrm{L}+3$, 如前文所述, H-3 和 H-2 分子轨道均为特殊对 $P$ 与辅助细菌叶绿素 $\mathrm{B}_{\mathrm{L}}$ 的贡献, $\mathrm{L}+3$ 分子轨道主要为色素 $\mathrm{B}_{\mathrm{L}}$ 的贡献, 因此, $5^{1} A$ 激发态主要由辅助细菌叶绿素 $\mathrm{B}_{\mathrm{L}}$ 的电子激发和 $\mathrm{P} \rightarrow \mathrm{B}_{\mathrm{L}}$ 的 $\mathrm{CT}$ 组成. $6^{1} A$ 激发态的跃迁相关组态主要是 $\mathrm{H}-4 \rightarrow \mathrm{L}+2, \mathrm{H}-4$ 和 $\mathrm{L}+2$ 分子轨道主要来自色素 $\mathrm{H}_{\mathrm{M}}$ 的 贡献, 因此, 我们将 $6^{1} A$ 激发态归属为 $\mathrm{H}_{\mathrm{M}}$ 的电子激 发. 参见图 2 和 3.

Rps. viridis光合反应中心是个较为复杂的生物体 系，因此其激发态光谱的归属也就成了一个争议性 较强的研究课题. 为了能更好的对实验吸收光谱进 行解释和归属, 本文同时考虑了实验 $\mathrm{CD}$ 光谱 ${ }^{[21]}$ 进行 对比研究. 图 2 为ZINDO计算的UV光谱与实验吸收 光谱 ${ }^{[20]}$ 的比较, 图 3 为ZINDO计算的 CD 光谱和实验 的 CD光谱 ${ }^{[21]}$ 的比较, 具体计算数据列于表 2 和表 3 . SAC-CI ${ }^{[13]}$ 的计算结果及其归属也列入表 3 做比较. 由图 2 可见, 实验吸收光谱在 $1.15 \sim 1.7 \mathrm{eV}$ 范围内主要 有 5 个强吸收峰, 从 $\mathrm{I} \sim \mathrm{V}$, 虚线表示其归属.

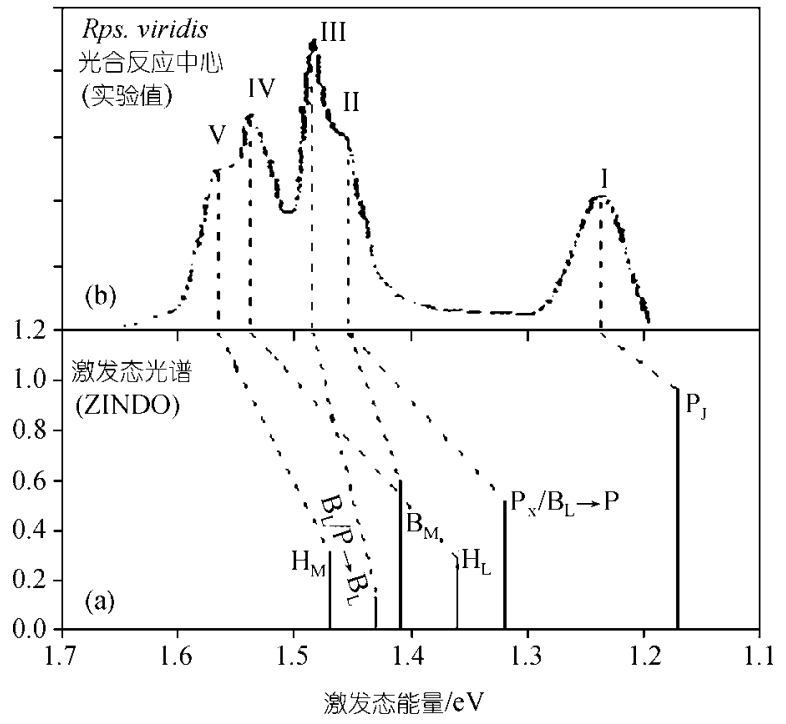

图 2 Rps. viridis 光合反应中心的吸收光谱 (a)实验吸收光谱 ${ }^{211}$; (b)ZINDO的理论计算光谱

峰I的实验峰值约为 $1.25 \mathrm{eV}$, 这与ZINDO计算的 $\mathrm{P}_{\mathrm{y}}\left(1^{1} A\right)$ 激发态的 $1.17 \mathrm{eV}$ 接近, 由图 3(c)中可以看到 $\mathrm{P}_{\mathrm{y}}\left(1^{1} A\right)$ 激发态与实验 $\mathrm{CD}$ 光谱中的第一个峰方向一致, 均为正值. 而且根据Shuvalov ${ }^{[21]}$ 等人的实验研究, 如 图 3(b)虚线所示, 当 $\mathrm{P}$ 被氧化成 $\mathrm{P}^{+}$后, 第一个峰消失, 因此本文确定图 3 中的第一个峰主要来自 $\mathrm{P}_{\mathrm{y}}\left(1^{1} A\right)$ 态 的贡献, 对应于实验吸收光谱(图 2)中的峰I. 这与 SAC-CI的归属一致, 但是ZINDO的结果似乎更接近 与实验值. 如表 3 所示, ZINDO的计算值比实验峰值 红移 $0.08 \mathrm{eV}$, 而SAC-CI模型分子的计算结果比实验 峰值红移 $0.16 \mathrm{eV}$.

峰 II 的实验峰值约为 $1.46 \mathrm{eV}$, 本文归属为 $\mathrm{P}_{\mathrm{x}} / \mathrm{B}_{\mathrm{L}}$ $\rightarrow \mathrm{P}\left(2^{1} A\right)$ 激发态 $(1.32 \mathrm{eV})$ 及 $\mathrm{B}_{\mathrm{M}}\left(4^{1} A\right)$ 激发态 $(1.41 \mathrm{eV})$ 


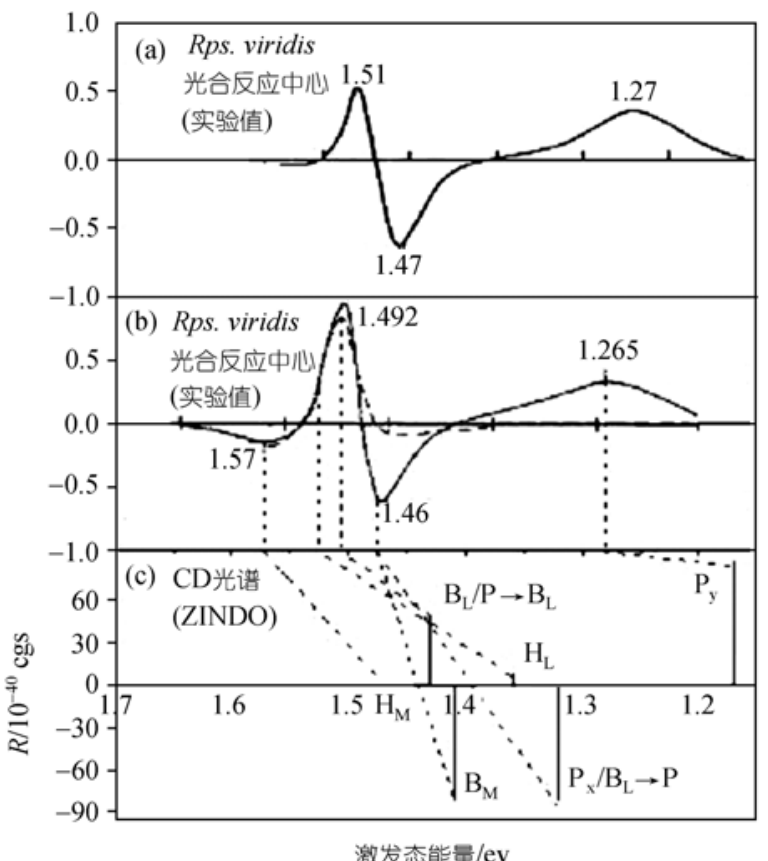

图 3 实验 $\mathrm{CD}$ 光谱和 $\mathrm{ZINDO}$ 计算光谱

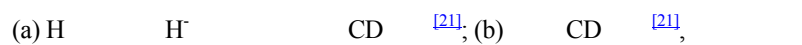
为 $\mathrm{P}$ 被氧化为 $\mathrm{P}^{+}$后测得的实验光谱; (c) ZINDO 计算的理论 $\mathrm{CD}$ 光谱

的贡献. 此峰在峰 III 右边, 两峰非常接近, 因此只根 据计算的激发态能量大小来进行归属是比较困难的, 需要借助 $C D$ 光谱的数据进行联合归属. 从图 3(c) 可 以看到, ZINDO 计算的 $\mathrm{P}_{\mathrm{x}} / \mathrm{B}_{\mathrm{L}} \rightarrow \mathrm{P}$ 激发态和 $\mathrm{B}_{\mathrm{M}}$ 激发态 的旋光强度均为负值, 这与实验 $\mathrm{CD}$ 光谱(图 3)的第 二个峰方向一致, 且从图 3(b) 的虚线可以看到当 $\mathrm{P}$ 被氧化成 $\mathrm{P}^{+}$后, 第二个峰的强度几乎消失, 但并没有 完全消失, 说明此峰绝大部分为发色团 $\mathrm{P}$ 的贡献, 但 还可能有发色团 B 或 $H$ 的贡献. 此外, 如图 3(a)所示, 当发色团 $\mathrm{H}$ 被还原为 $\mathrm{H}^{-}$后, 第二个峰并没有太大的 变化, 因此本文将 $\mathrm{P}_{\mathrm{x}} / \mathrm{B}_{\mathrm{L}} \rightarrow \mathrm{P}$ 激发态归属为第二个峰 的主峰, $\mathrm{B}_{\mathrm{M}}$ 激发态归属为侧峰, 此峰对应于实验吸收 光谱(图 2)中的峰 II. SAC-CI 将 $\mathrm{P}_{\mathrm{x}}$ 归属为峰 II, 将 $\mathrm{B}_{\mathrm{M}}$ 归属为峰 III, 这很难解释为什么 $\mathrm{P}$ 被氧化成 $\mathrm{P}^{+}$后峰 II 没有完全消失.

根据Shuvalov ${ }^{[21]}$ 等人的实验研究，当色素 $\mathrm{H}$ 被还 原为 $\mathrm{H}^{-}$时, 实验 $\mathrm{CD}$ 光谱(图 3(a))中第三个峰的强度 减弱了很多, 而第四个峰则完全消失, 由此可以推断 出第四个峰主要来自色素 $\mathrm{H}$ 的贡献, 第三个峰除了 $\mathrm{H}$
的贡献外还应有其他色素分子的贡献. 在此能量区 域, ZINDO的计算结果 (图 3(c)) 显示 $\mathrm{H}_{\mathrm{L}}$ 和 $\mathrm{B}_{\mathrm{L}} / \mathrm{P} \rightarrow \mathrm{B}_{\mathrm{L}}$ 激 发态的旋光度为正, $\mathrm{H}_{\mathrm{M}}$ 激发态的旋光度为负, $\mathrm{H}_{\mathrm{M}}$ 态的 激发能大于 $\mathrm{H}_{\mathrm{L}}$ 态的激发能，因此，我们将 $\mathrm{H}_{\mathrm{L}}$ 态归属 为第三个峰的侧峰, $\mathrm{B}_{\mathrm{L}} / \mathrm{P} \rightarrow \mathrm{B}_{\mathrm{L}}$ 态归属为第三个峰的主 峰, $\mathrm{H}_{\mathrm{M}}$ 态归属为第四个峰. 对应于实验吸收光谱(图 2(a)), 峰III主要来自于 $\mathrm{B}_{\mathrm{L}} / \mathrm{P} \rightarrow \mathrm{B}_{\mathrm{L}}$ 激发态的贡献, 这与 SAC-CI归属(来自于 $\mathrm{B}_{\mathrm{L}}$ 和 $\mathrm{B}_{\mathrm{M}}$ 的贡献)不同. 在此说明 一点, 我们认为 $\mathrm{H}_{\mathrm{L}}$ 态的激发能应高于 $\mathrm{B}_{\mathrm{L}} / \mathrm{P} \rightarrow \mathrm{B}_{\mathrm{L}}$ 态的 激发能, ZINDO方法可能低估了 $\mathrm{H}_{\mathrm{L}}$ 激发态的能量. 因 此, 本文将峰IV 和峰 $\mathrm{V}$ 分别归属为 $\mathrm{H}_{\mathrm{L}}$ 和 $\mathrm{H}_{\mathrm{M}}$ 的激发态 贡献, 这与SAC-CI的归属一致. 本文的归属合理地 解释了当 $\mathrm{P}$ 被氧化为 $\mathrm{P}^{+}$时, $\mathrm{CD}$ 光谱的第三个峰会微弱 的降低, 主要原因是该峰有 $\mathrm{P} \rightarrow \mathrm{B}_{\mathrm{L}}$ 电荷转移的贡献. 此外, $\mathrm{P} \rightarrow \mathrm{B}_{\mathrm{L}}$ 电荷转移说明特殊对 $\mathrm{P}$ 与辅助细菌叶绿素 $\mathrm{B}_{\mathrm{L}}$ 之间有着较强的相互作用, 容易发生电荷转移, 这 在某种程度上解释了特殊对 $\mathrm{P}$ 受到光激发后电子只沿 着 $\mathrm{L}$ 分枝传递.

从表 2 可以看到, INDO/S 计算的光谱较实验峰 值蓝移, ZINDO 的能量则红移. ZINDO 计算的 $\mathrm{P}_{\mathrm{x}}, \mathrm{B}_{\mathrm{M}}$, $\mathrm{B}_{\mathrm{L}}$ 和 $\mathrm{H}_{\mathrm{M}}$ 态较为精确, 其能量与实验值只相差约 $0.08 \mathrm{eV}$; 而 $\mathrm{P}_{\mathrm{y}}$ 和 $\mathrm{H}_{\mathrm{L}}$ 能量误差较大, 约为 $0.13 \mathrm{eV}$. $\mathrm{INDO} / \mathrm{S}$ 计算的各激发态能量误差都在 $0.15 \mathrm{eV}$ 左右, 相比而言 ZINDO 的结果较为合理, 因此, 这里我们 只详细讨论 ZINDO 的计算结果.

从表 2 还可以看到, ZINDO 计算的 $2^{1} A(\mathrm{P} \rightarrow \mathrm{P}$ $/ B_{L} \rightarrow P$ )态中 $P \rightarrow P$ 和 $B_{L} \rightarrow P$ 跃迁的相关组态系数分别 为 0.35 和 0.33 , 说明 $\mathrm{P} \rightarrow \mathrm{P}$ 和 $\mathrm{B}_{\mathrm{L}} \rightarrow \mathrm{P}$ 的电子跃迁都对 $2^{1} A$ 态有较大贡献; $5^{1} A\left(B_{L} \rightarrow B_{L} / P \rightarrow B_{L}\right)$ 态中 $B_{L} \rightarrow B_{L}$ 和 $\mathrm{P} \rightarrow \mathrm{B}_{\mathrm{L}}$ 跃迁的相关组态系数分别是 0.40 和 0.39 (如表 $2)$, 说明 $\mathrm{B}_{\mathrm{L}} \rightarrow \mathrm{B}_{\mathrm{L}}$ 和 $\mathrm{P} \rightarrow \mathrm{B}_{\mathrm{L}}$ 的电子跃迁都对 $5^{1} A$ 态有较 大贡献. 因此, 这两个激发态既有色素分子自身电子 激发的贡献, 也有 $\mathrm{B}_{\mathrm{L}}$ 与 $\mathrm{P}$ 之间的电荷转移的贡献. 而 基于单个分子模型的 SAC-CI 研究只能得到了 $B_{L}$ 与 $P$ 自身电子激发的贡献. 这种差别的体现是本文研究 意义的所在. ZINDO 对实验吸收光谱峰 II 的归属为 $\mathrm{P}_{\mathrm{x}} / \mathrm{B}_{\mathrm{L}} \rightarrow \mathrm{P}$ 态和 $\mathrm{B}_{\mathrm{M}}$ 态的贡献, 而 SAC-CI 将其归属为 $\mathrm{P}_{\mathrm{x}}$ 的贡献. SAC-CI 的上述归属很难解释当 $\mathrm{P}$ 被氧化 后, 实验 $\mathrm{CD}$ 光谱(图 $3 b$ )的第二个峰并没有完全消失. 
对于实验吸收峰 III(图 2(a)), ZINDO 归属为 $\mathrm{B}_{\mathrm{L}} / \mathrm{P} \rightarrow \mathrm{B}_{\mathrm{L}}$ 态的贡献,而 SAC-CI 归属为 $B_{M}$ 和 $B_{L}$ 态的贡献. ZINDO 的归属可以较好的解释 $\mathrm{P}$ 被氧化后实验 $\mathrm{CD}$ 光谱的第三个峰(图 3(b))的强度有一定的减小, 而 SAC-CI 的归属却不能解释(详见表 3).

为了考查 SAC-CI 研究中所采用的单个分子模型 对激发态光谱性质的影响, 下面我们比较基于两种 不同分子模型(分子簇和单个分子)的 ZINDO 理论 CD 光谱(如图 4 所示).

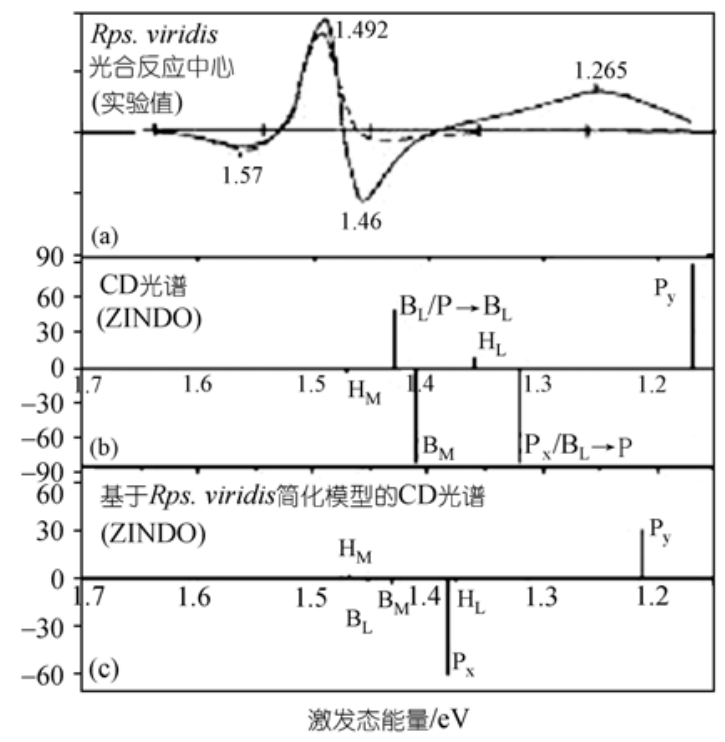

图 4

(a) 实验 $\mathrm{CD}$ 光谱 ${ }^{[21]}$; (b)基于分子簇模型的ZINDO计算 $\mathrm{CD}$ 光谱;

基于单个分子模型(SAC-CI研究采用)的ZINDO计算CD光谱

从图 4 中(b)和(c)的比较可以看到, 基于单个分 子模型计算的 $\mathrm{CD}$ 光谱激发能发生了一些蓝移, 如 $\mathrm{P}_{\mathrm{y}}$ 和 $\mathrm{P}_{\mathrm{x}}$ 这两个激发态分别蓝移了约 0.04 和 $0.06 \mathrm{eV}$, 虽 然从激发能大小的角度来说更接近实验值, 但是其 它一些激发态的性质和旋光度发生了较大的变化. 由于采用的单个分子模型不能考虑色素分子间的相 互作用, 因此在此模型下不可能发现 $\mathrm{B}_{\mathrm{L}}$ 和 $\mathrm{P}$ 色素分 子间的电荷转移; 此外, $\mathrm{H}_{\mathrm{L}}$ 和 $\mathrm{B}_{\mathrm{L}}$ 态的旋光度均变成 了负值, 而 $\mathrm{H}_{\mathrm{M}}$ 态的旋光度则变为正值, 变化后的 理论结果很难对实验 $\mathrm{CD}$ 光谱进行合理归属. 由此 可见, 计算模型的选取对激发态光谱的计算有较大 的影响.

\section{2 氨基酸残基对光谱的影响}

除色素分子间的相互作用外，周围氨基酸残基 分子对激发态光谱的影响也是一个不可忽视的因素. 下面我们考虑了距光合反应中心色素分子簇 $2.5 \AA$ 范 围内的氨基酸残基(主要是组氨酸, 如图 1 所示)的作 用, 计算结果列于表 2 .

考虑了组氨酸的作用后, ZINDO 计算的 $\mathrm{P}_{\mathrm{y}}$ 态的 激发能蓝移了 $0.05 \mathrm{eV}$, 更接近于实验值 $1.25 \mathrm{eV} ; \mathrm{B}_{\mathrm{M}}$ 态的激发能红移了 $0.03 \mathrm{eV}$; 对 $\mathrm{P}_{\mathrm{x}} / \mathrm{B}_{\mathrm{L}} \rightarrow \mathrm{P}, \mathrm{B}_{\mathrm{L}}$ 和 $\mathrm{H}_{\mathrm{M}}$ 态 几乎没有影响; $\mathrm{H}_{\mathrm{L}}$ 态红移了 $0.01 \mathrm{eV}$. 由此可见, 环境 氨基酸残基分子对光合反应中心分子簇的电子激发 态产生一定的影响. 氨基酸残基对含有色素 $\mathrm{P}$ 和 $\mathrm{B}$ 贡 献的光谱影响较大, 从表 2 的比较中可以发现, 考虑 氨基酸作用使含 $\mathrm{P}$ 贡献的激发态的能量增大, 而含 $\mathrm{B}$ 贡献的激发态能量减小, 即 $\mathrm{P}_{\mathrm{y}}$ 的能量发生了一定蓝 移, 而 $\mathrm{B}_{\mathrm{M}}$ 和 $\mathrm{B}_{\mathrm{L}}$ 的能量都有一定的红移. 由于 $\mathrm{H}-2$ 分 子轨道是由 $P$ 和 $B_{L}$ 的贡献组成，所以 $P_{x} / B_{L} \rightarrow P$ 态的 能量变化较小. 由此可以看出环境氨基酸残基对激 发态的计算有一定的影响, 考虑了蛋白质环境效应 的计算光谱会更好地与实验光谱相吻合.

\section{3 分子结构对激发态光谱的影响}

最近, Reimers等 ${ }^{[27]}$ 采用 $\mathrm{QM} / \mathrm{MM}$ 方法(色素分子 簇采用 AM1 方法, 周围的氨基酸采用UFF力场)对Rps. viridis光合反应中心的分子结构进行了优化，我们基 于这个优化结构进行了激发态光谱的初步研究. 由 于篇幅有限本文只作简要介绍(见图 5), 具体计算和 讨论细节将在另一篇系列文章中介绍.

图 5 中, (a)为实验吸收光谱 ${ }^{[20]}$, (b)为基于晶体结 构所计算的UV光谱, (c)为基于Reimers ${ }^{[27]}$ 优化的结构 所计算的UV光谱. 表 3 列出了基于晶体结构的 ZINDO(1)计算结果和基于Reimers优化构型的ZIN$\mathrm{DO}(3)$ 计算结果. 如图 5 所示, Reimers优化结构使激 发态光谱发生了一定的变化, $\mathrm{B}_{\mathrm{M}}$ 和 $\mathrm{B}_{\mathrm{L}}$ 激发态的能量 变化较小, $\mathrm{B}_{\mathrm{M}}$ 激发态的能量保持不变, $\mathrm{B}_{\mathrm{L}}$ 激发态约有 $0.1 \mathrm{eV}$ 的红移. 而 $\mathrm{P}$ 和 $\mathrm{H}$ 激发态的能量变化较大, $\mathrm{P}_{\mathrm{y}}$ 激 发态蓝移了近 $0.4 \mathrm{eV}, \mathrm{P}_{\mathrm{x}}$ 激发态有近 $0.3 \mathrm{eV}$ 的蓝移, $\mathrm{H}_{\mathrm{L}}$ 激发态蓝移了 $0.73 \mathrm{eV}, \mathrm{H}_{\mathrm{M}}$ 激发态蓝移了 $0.66 \mathrm{eV}$. 由 此可见, Reimers优化结构使 $\mathrm{P}$ 与 $\mathrm{H}$ 发色团的结构 
表 3 各种计算方法的激发态能量及振子强度与实验值的比较

\begin{tabular}{|c|c|c|c|c|c|c|c|c|c|}
\hline \multirow{2}{*}{ 发色团 } & \multirow{2}{*}{ 激发态 } & \multicolumn{2}{|c|}{ ZINDO(1) $)^{\mathrm{a})}$} & \multicolumn{2}{|c|}{$\operatorname{ZINDO}(3)^{\mathrm{b})}$} & \multicolumn{2}{|c|}{ SAC-CI ${ }^{[13]}$} & \multicolumn{2}{|c|}{ 实验值 ${ }^{[20,21]}$} \\
\hline & & 激发能/eV & 振子强度 & 激发能/eV & 振子强度 & 激发能/eV & 振子强度 & 激发能/eV & 峰 \\
\hline $\mathrm{P}_{\mathrm{y}}$ & $1^{1} \mathrm{~A}$ & 1.17 & 0.951 & 1.54 & 0.364 & $1.41(\mathrm{I})^{\mathrm{c})}$ & $0.607 \mathrm{P}_{\mathrm{y}}$ & 1.25 & I \\
\hline $\mathrm{P}_{\mathrm{x}} / \mathrm{B}_{\mathrm{L}} \rightarrow \mathrm{P}$ & $2^{1} \mathrm{~A}$ & 1.32 & 0.502 & 1.61 & 0.132 & $1.61(\mathrm{II})$ & $0.090 \mathrm{P}_{\mathrm{x}}$ & 1.46 & II \\
\hline $\mathrm{H}_{\mathrm{L}}$ & $3^{1} A$ & 1.36 & 0.276 & 2.09 & 0.335 & 1.59 (IV) & $0.323 \mathrm{H}_{\mathrm{L}}$ & 1.49 & IV \\
\hline $\mathrm{B}_{\mathrm{M}}$ & $4^{1} \mathrm{~A}$ & 1.41 & 0.589 & 1.41 & 0.491 & $1.35(\mathrm{III})$ & $0.363 \mathrm{~B}_{\mathrm{M}}$ & 1.46 & II \\
\hline $\mathrm{B}_{\mathrm{L}} / \mathrm{P} \rightarrow \mathrm{B}_{\mathrm{L}}$ & $5^{1} A$ & 1.43 & 0.126 & 1.35 & 0.783 & $1.45(\mathrm{III})$ & $0.406 \mathrm{~B}_{\mathrm{L}}$ & 1.49 & III \\
\hline $\mathrm{H}_{\mathrm{M}}$ & $6^{1} \mathrm{~A}$ & 1.47 & 0.305 & 2.13 & 0.357 & $1.75(\mathrm{~V})$ & $0.283 \mathrm{H}_{\mathrm{M}}$ & 1.57 & V \\
\hline
\end{tabular}

a) 基于晶体结构且未考虑氨基酸残基的作用的 ZINDO 计算; b)基于 Reimers 等人用 QM/MM 方法优化的结构且未考虑氨基酸残基的作用的 ZINDO 计算; c) 括号中的编号为 SAC-CI 的能量所对应的实验峰

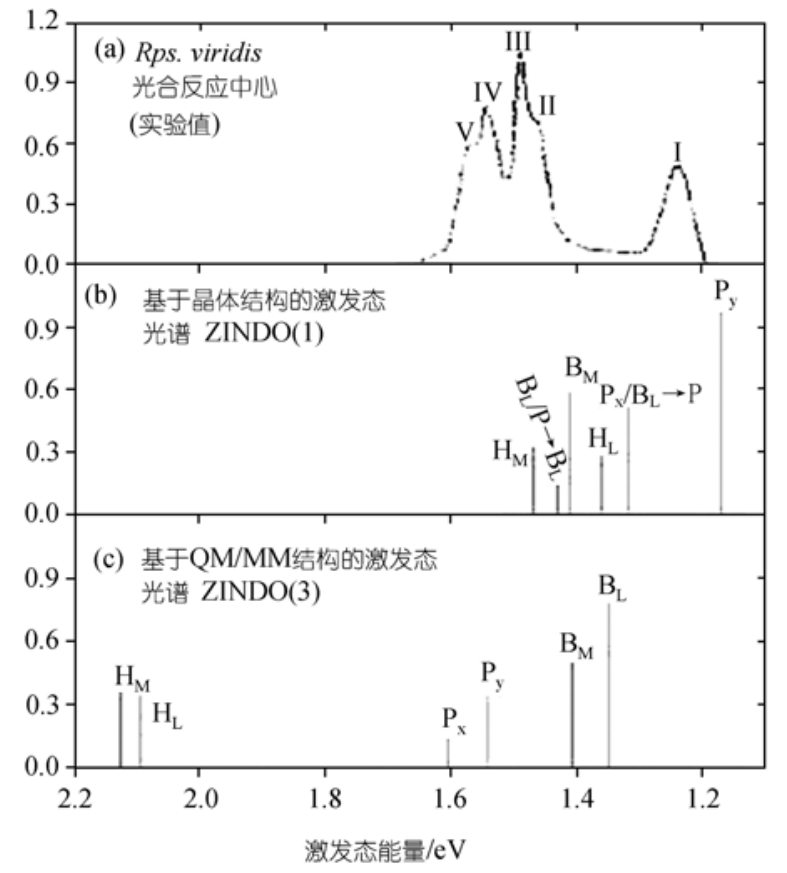

图 5 基于不同结构的理论激发态光谱与 实验吸收光谱的比较

(a) 实验吸收光谱 ${ }^{[20]}$; (b) 基于晶体结构的ZINDO计算UV吸收光谱; (c) 基于Reimers ${ }^{[27]}$ 优化结构的ZINDO计算UV吸收光谱

发生了较大的变化, 分子结构的变化对激发态能量 产生了一定的影响. 基于晶体结构计算的激发态光 谱与实验光谱吻合较好, 可以对实验光谱进行较为 合理的解释和归属; 基于 Reimers 优化结构计算的激 发态光谱与实验光谱相差较远, 因此对此类光合反 应中心分子结构的合理优化是一项复杂而重要的工 作. 接下来我们将对整个 Rps. viridis 光合反应中心进 行分子动力学模拟(MD)和更高理论级别的量子化学
计算, 并进行激发态理论光谱的计算和比较研究, 以 期寻找出更为合理的 RSRC 结构模型.

\section{3 结论}

(i) 通过本文的比较研究, 再次验证了 ZINDO 和 INDO/S 两种半经验分子轨道方法对低能带激发态的 预测和归属具备了一定的精度. 对于一些较大的生 物分子体系, 目前运用 TDDFT, SAC-CI 等高精度 Post-SCF 理论方法进行整个 PSRC 分子簇的计算是相 当困难的, 而 ZINDO 和 INDO $/ \mathrm{S}$ 方法可以较为有效 的对上述体系低能带区域的实验光谱进行合理的归 属. 考虑了色素分子间相互作用、氨基酸残基影响的 ZINDO 的理论计算较为合理地解释了实验光谱, 基 于此, 我们重新归属了实验吸收光谱和 $\mathrm{CD}$ 光谱.

(ii) 通过对紫色细菌光合反应中心一系列的计 算研究发现，色素之间的作用、周围氨基酸残基与色 素分子簇的作用、光合反应中心的特殊结构等对光谱 性质都有较为明显的影响.

(iii) 考虑了色素分子间相互作用的 ZINDO 计算 表明: $2^{1} A\left(\mathrm{P}_{\mathrm{x}} / \mathrm{B}_{\mathrm{L}} \rightarrow \mathrm{P}\right)$ 激发态和 $5^{1} A\left(\mathrm{~B}_{\mathrm{L}} / \mathrm{P} \rightarrow \mathrm{B}_{\mathrm{L}}\right)$ 激发态既 有色素分子自身电子激发的贡献, 也有 $\mathrm{B}_{\mathrm{L}}$ 和 $\mathrm{P}$ 色素 分子间的电荷转移的贡献(详见表 2). 这说明特殊对 $\mathrm{P}$ 与辅助细菌叶绿素 $\mathrm{B}_{\mathrm{L}}$ 之间有较强的相互作用, 它 们之间容易发生电荷转移, 因此某种程度上解释了 为什么特殊对 $\mathrm{P}$ 受到光激发后电子转移只沿着 $\mathrm{L}$ 分支 向下传递的特异性.

致谢 作者十分感谢日本京都大学工学部合成生物 化学系 Nakatsuji 教授提供的部分计算资源和富有建 
设性的讨论.

\section{参考 文 献}

1 Deisenhofer J, Epp O, Miki K, et al. Structure of the protein subunits in the photosynthetic reaction center of Rhodopseudomonas viridis at 3 A resolution. Nature, 1985, 318: 618 624 [DOI]

2 Deisenhofer J, Epp O, Sinning I, et al. Crystallographic refinement at $2.3 \AA$ resolution and refined model of the photosynthetic reaction centre from Rhodopseudomonas viridis. J Mol Biol, 1995, 246: 429 457 [DOI]

3 Horber J K H, Gobel W, Ogrodnik A, et al. Ln Antennas and Reaction Centers of Photosynthetic Bacteria. Berlin: Springer Verlag, 1985. 292

4 Hörber J K H, Göbel W, Ogrodnik A, et al. Time-resolved measurements of fluorescence from reaction centres of Rhodopseudomonas viridis and the effect of menaquinone reduction. FEBS Lett, 1986, 198: 268 272 [DOI]

5 Nakatsuji H. Cluster expansion of the wavefunction. Excited states. Chem Phys Lett, 1978, 59: 362 364

6 Nakatsuji H. Cluster expansion of the wavefunction. Electron correlations in ground and excited states by SAC (symmetry-adapted-cluster) and SAC CI theories. Chem Phys Lett, 1989, 67: 329 333 [DOI]

7 Nakatsuji H. Cluster expansion of the wavefunction. Calculation of electron correlations in ground and excited states by SAC and SAC CI theories. Chem Phys Lett, 1989, 67: 334 342 [DOI]

8 Bauernschmitt R, Ahlrichs R. Treatment of electronic excitations within the adiabatic approximation of time dependent density functional theory. Chem Phys Lett, 1996, 256: 454 464 [DOI]

9 Bauernschmitt R, Haser M, Treutler O, et al. Calculation of excitation energies within time-dependent density functional theory using auxiliary basis set expansions. Chem Phys Lett, 1997, 264: 573 578 [DOI]

10 Rubio M, Merchan M, Orti E. The internal rotational barrier of biphenyl studied with multiconfigurational second-order perturbation theory (CASPT2). Theor Chim Acta, 1995, 91: 17 29

11 Bartlett R J. Coupled-Cluster Theory: An Overview of Recent Developments. In: Yarkony D, ed. Modern Electronic Structure Theory, II. Singapore: World Scientific, 1995

12 Nakatsuji H, Hasegawa J, Ohkawa K. Excited states and electron transfer mechanism in the photosynthetic reaction center of Rhodopseudomonas viridis: SAC-CI study. Chem Phys Lett, 1998, 296: 499 504 [DOI]

13 Hasegawa J, Ohkawa K, Nakatsuji H. Excited states of the photo- synthetic reaction center of Rhodopseudomonnas viridis: SAC-CI study. J Phys Chem B, 1998, 102: 10410 10419 [DOI]

14 Dage Sundholm. A density-functional-theory study of bacteriochlorophyll b. Phys Chem Chem Phys, 2003, 5: 4265 4271 [DOI]

15 Hasegawa J, Ozeki Y, Ohkawa K, et al. Theoretical study of the excited states of chlorin, bacteriochlorin, pheophytin a, and chlorophyll a by the SAC/SAC-CI method. J Phys Chem B, 1998, 102: 1320 1326 [DOI]

16 Sundholm D. Density functional theory calculations of the visible spectrum of chlorophyll $a$. Chem Phys Lett, 1999, 302: 480 484 [DOI]

17 Sundholm D. Comparison of the electronic excitation spectra of chlorophyll a and pheophytin a calculated at density functional theory level. Chem Phys Lett, 2000, 317: 545 552 [DOI]

18 Pople J A, Beveridge D, Dobosh P. Approximate self-consistent molecular-orbital theory. V. Intermediate neglect of differential overlap. J Chem Phys, 1967,47: 2026 2033

19 Zerner M C, Lowe G H, Kirchner R F, et al. An intermediate neglect of differential overlap technique for spectroscopy of transition-metal complexes. Ferrocene. J Am Chem Soc, 1980, 102: 589 599 [DOI]

20 Breton J. Orientation of the chromophores in the reaction center of Rhodopseudomonas viridis. Comparison of low-temperature linear dichroism spectra with a model derived from X-ray crystallography. Biochim Biophys Acta, 1985, 810: 235 245

21 Shuvalov V A, Asadov A A. Arrangement and interaction of pigment molecules in reaction centers of Rhodopseudomonas viridis. Biochim Biophys Acta, 1979, 545: 296 308

22 Frisch M J, Trucks G W, Schlegel H B, et al. Gaussian 03, Revision B.03. Pittsburgh: Gaussian, Inc, 2003.

23 Stewart J J P. Optimization of parameters for semi-empirical methods I-Method. J Comp Chem, 1989, 10: 209 220 [DOI]

24 Rappé K, Casewit C J, Colwell K S, et al. UFF, a full periodic table force field for molecular mechanics and molecular dynamics simulations. J Am Chem Soc, 1992, 114: 10024 10035 [DOI]

25 Pople J A, Beveridge D L. Approximate Molecular Orbital Theory. New York: MacGraw-Hill, 1970

26 Ren Y L, Wan J, Liu J J, et al. A theoretical comparison study on vertical excited states of free-base porphin. Acta Phys Chim Sin, 2004, 20: 1089 1092

27 Hughes J M, Michael C, Reimers J R, et al. Modeling the bacterial photosynthetic reaction center. 4 . The structural, electrochemical, and hydrogen-bonding properties of 22 mutants of rhodobacter sphaeroides. J Am Chem Soc, 2001, 123: 8550 8563 [DOI] 